\title{
Nano-fried-eggs: Structural, optical, and magnetic characterization of physically prepared iron-silver nanoparticles
}

DOI:

10.1007/s12274-018-2125-6

\section{Document Version}

Accepted author manuscript

Link to publication record in Manchester Research Explorer

Citation for published version (APA):

Ramade, J., Troc, N., Boisron, O., Pellarin, M., Lebault, M., Cottancin, E., Oiko, V. T. A., Gomes, R. C., Rodrigues, V., \& Hillenkamp, M. (2018). Nano-fried-eggs: Structural, optical, and magnetic characterization of physically prepared iron-silver nanoparticles. Nano Research, 1-12. https://doi.org/10.1007/s12274-018-2125-6

\section{Published in:}

Nano Research

\section{Citing this paper}

Please note that where the full-text provided on Manchester Research Explorer is the Author Accepted Manuscript or Proof version this may differ from the final Published version. If citing, it is advised that you check and use the publisher's definitive version.

\section{General rights}

Copyright and moral rights for the publications made accessible in the Research Explorer are retained by the authors and/or other copyright owners and it is a condition of accessing publications that users recognise and abide by the legal requirements associated with these rights.

\section{Takedown policy}

If you believe that this document breaches copyright please refer to the University of Manchester's Takedown Procedures [http://man.ac.uk/04Y6Bo] or contact uml.scholarlycommunications@manchester.ac.uk providing relevant details, so we can investigate your claim.

\section{OPEN ACCESS}




\title{
Nano-fried-eggs: Structural, Optical and Magnetic Characteri- zation of Physically Prepared Iron-Silver Nanoparticles
}

\author{
Julien Ramade ${ }^{1}$, Nicolas Troc ${ }^{1}$, Olivier Boisron ${ }^{1}$, Michel Pellarin ${ }^{1}$, Marie-Ange Lebault ${ }^{1}$, Emmanuel Cot- \\ tancin $^{1}$, Vitor T.A. Oiko ${ }^{2}$, Rafael Cabreira Gomes ${ }^{2}$, Varlei Rodrigues ${ }^{2}$, Matthias Hillenkamp ${ }^{1,2}(\bowtie)$ \\ ${ }^{1}$ Univ. Lyon, Université Claude Bernard Lyon 1, CNRS, UMR5306, Institut Lumière Matière, F-69622, Villeurbanne, France. \\ ${ }^{2}$ Instituto de Física Gleb Wataghin, UNICAMP, CP 6165, 13083-970 Campinas, SP, Brazil.
}

Received: day month year / Revised: day month year / Accepted: day month year (automatically inserted by the publisher)

(C) Tsinghua University Press and Springer-Verlag Berlin Heidelberg 2011

\begin{abstract}
The prospect of combining both magnetic and plasmonic properties in one single nanoparticle promises both valuable insights on the properties of such systems from a fundamental point of view as well as numerous possibilities in technological applications. The combination of two of the most prominent metallic candidates, iron and silver, has, however, presented a lot of experimental difficulties because of their thermodynamic properties impeding miscibility or even coalescence. Here we present the thorough characterization of physically prepared $\mathrm{Fe}_{50} \mathrm{Ag}_{50}$ nanoparticles embedded in carbon and silica matrices by electron microscopy, optical spectroscopy, magnetometry and synchrotron-based x-ray spectroscopy. Iron and silver segregate completely into structures resembling fried eggs with a nearly spherical, crystallized silver part surrounded by an amorphous structure of iron carbide or oxide, depending on the environment of the particles. Consequently the particles display both plasmonic absorption corresponding to the silver nanospheres in an oxide environment as well as a reduced but measurable magnetic response. The suitability of such nanoparticles for technological applications is discussed in view of their high chemical reactivity with their environment.
\end{abstract}

\section{KEYWORDS}

nanoalloy, bimetallic nanoparticles, iron, silver, optical spectroscopy, magnetometry

Address correspondence to matthias.hillenkamp@univ-lyon1.fr 


\section{Introduction}

Metal nanoparticles have attracted an ever greater attention over the last decades in both fundamental and applied research because of the great variability and tunability of their physical and chemical properties through their composition, size and shape dependence. Namely purely nanoscopic effects such as the localized surface plasmon resonance [1] and superparamagnetism [2] are the subject of intense investigations. This motivation is even greater when two or more metals are combined at the nanoscale and when composition effects play an additional, often decisive role $[3,4,5]$. A whole immense playground of fascinating new properties and possibilities opens up, with e.g. efficient nano-catalysts made of immiscible metals [6] or theranostics applications [7]. The complexity of such systems requires, on the other hand, a detailed understanding of the underlying effects and interplays for the efficient design of nanosystems with new and tunable properties through size, shape, composition, and atomic structure of multi-metallic clusters.

Combining ferromagnetic transition metals and noble metals in nanoparticles is of particular interest in many aspects. The presence of plasmonic and magnetic properties in the same particle allows studying the same systems from several complementary angles. Furthermore such nanosystems promise potential use e.g. in magneto-plasmonics $[8,9]$. Applications for such multifunctional magnetic NPs are envisaged in biomedical applications [10] or for surface enhanced Raman spectroscopy (SERS) [11], as contrast agents or for information technology via magneto-optical switching [12].

One ideal candidate for a magneto-plasmonic system is $\mathrm{FeAg}$. Iron has a very high saturation magnetization, four times higher than e.g. one of the most abundant oxides, magnetite $\mathrm{Fe}_{3} \mathrm{O}_{4}$, whereas $\mathrm{Ag}$ has a four times higher extinction coefficient than $\mathrm{Au}$ at the surface plasmon resonance [13]. The extremely high affinity of iron towards oxidation has, however, hindered the fabrication of purely metallic FeAg nanoparticles and most studies are focused on the fabrication and study of particles combining silver with iron oxides $[9,14]$ as well as their applications in e.g. biomedicine [15], theranostics [16], SERS [17], catalysis and imaging [18]. The question whether and how it is possible to stabilize FeAg nanoparticles without oxidation, as it is e.g. possible for FePt [19] is important but unanswered today.

Fe and Ag are completely immiscible in the bulk and liquid phases [20] and no stable substituting alloy, where $\mathrm{Ag}$ on a bcc site in Fe or vice versa Fe on a fcc site in $\mathrm{Ag}$, has been observed in the bulk phase. The thermodynamically stable state accessible by thermal treatment consists of pure iron precipitates in a silver matrix [21, 22]. Extended X-ray Absorption Fine Structure (EXAFS) investigations of granular FeAg layers have shown that the as prepared state is a metastable solid solution while annealing leads to precipitation of iron into bcc structures [23].

Combining two immiscible metals in one nanoparticle is difficult. In pure FeAg particles we expect an iron core surrounded by a silver shell, given the difference in surface tension of the two metals [24]. In chemical synthesis typically segregated core-shell or dumbbellshaped structures are obtained $[25,9,26]$. In the few reported cases of chemically prepared $\mathrm{Fe}-\mathrm{Ag}$ nanoparticles the core-shell structure prevails [13, 27, 28]. Both Fe@Ag and Ag@Fe particles have been obtained, showing that the fabrication sequence and presence of surfactants determine the structure. Such particles have already been tested for catalysis [29] and antimicrobial treatment [30]. A fabrication technique combining physical and chemical approaches is to generate metal vapor by laser vaporization in a liquid containing functionalizing molecules, typically thiols. Ramified structures termed nanotruffles were observed for the Fe-Ag system [31] and the use of this fabrication method for magnetically assembled SERS substrates has been reported [32].

This overview clearly demonstrates the complexity one should expect concerning both the geometric structure of such nanoparticles as well as their physical and chemical properties. Before choosing adequate candidate systems for magneto-optical or -plasmonic studies and applications a thorough study based on complementary experimental and theoretical techniques is mandatory. In this article we present such an exhaustive study for FeAg nanoparticles fabricated by laser vaporization in the gas phase, thus without chemical surfactants. The only mention in the literature of the use of this technique for the fabrication of small clusters combining iron and silver has demonstrated its feasibility but the stoichiometry was shown to be very complex, reflecting the unfavorable 
Fe-Ag bonding [33]. We characterized our particles with methods such as electron microscopy, optical spectroscopy, magnetometry and synchrotron-based $x$-ray spectroscopy. The consistent combination of complementary techniques allows discriminating between different structural possibilities and physical effects in a system as complex as iron-silver and thus permits evaluating the suitability of our particles for further studies and applications.

\section{Experimental}

\subsection{Sample preparation}

Nanoparticles are produced in a conventional laser vaporization source [34]. The second harmonic of a nanosecond $\mathrm{Nd}^{3+}$ :YAG pulsed laser is focused on the surface of a metallic target generating an atomic plasma in the presence of a continuous flow of helium gas. The atomic vapor is rapidly cooled down by collisions with the inert gas (static pressure of a few tens of mbars) which induces the nucleation and growth of small metal clusters (2-6 nm in diameter). The particle-gas mixture expands into vacuum through a conical nozzle to form of a supersonic beam towards a deposition chamber. Bimetallic clusters are essentially produced from an $\mathrm{Fe}_{0.5} \mathrm{Ag} 0.5$ bi-metallic target $(50 \%-50 \%$ atomic composition, Neyco sa., France). The study of many miscible and immiscible metals elaborated this way has shown that the mean stoichiometry in the nanoparticles is the same as in the target rods $[35,36]$.
The nanoparticles are then deposited under soft-landing conditions for either electron microscopy, magnetic or optical experiments. For electron microscopy ultrathin carbon films (Ted Pella Inc.) are used and the particles are either left uncapped or protected by sandwiching them between two evaporated layers of several nanometers each, in our case amorphous carbon or silica. For ensemble measurements the particles are co-deposited together with the protective matrix (amorphous carbon or silica) onto adequate substrates which are fused silica for optical experiments or silicon wafers for magnetism and synchrotron experiments. For optical and magnetic measurements the particle density was kept below $\sim 1$ vol.\% in order to avoid inter-particle interactions, the sample for XMCD was more concentrated ( $\sim 5$ vol.\%) to increase the signal/noise ratio. No post-fabrication heat treatment has been performed on the samples discussed here.

Two types of matrices have been used to isolate the nanoparticles and protect them from thermal and chemical degradation: amorphous carbon for magnetic measurements and silica as a transparent oxide for optical measurements. Both systems have furthermore been investigated in electron microscopy. Amorphous carbon has been shown to be an excellent matrix for coalescence-free annealing [37, 38, 39] of mono- and bi-metallic clusters. At the same time even a very thin layer of carbon of the order of $5 \mathrm{~nm}$ thickness is generally sufficient for complete protection

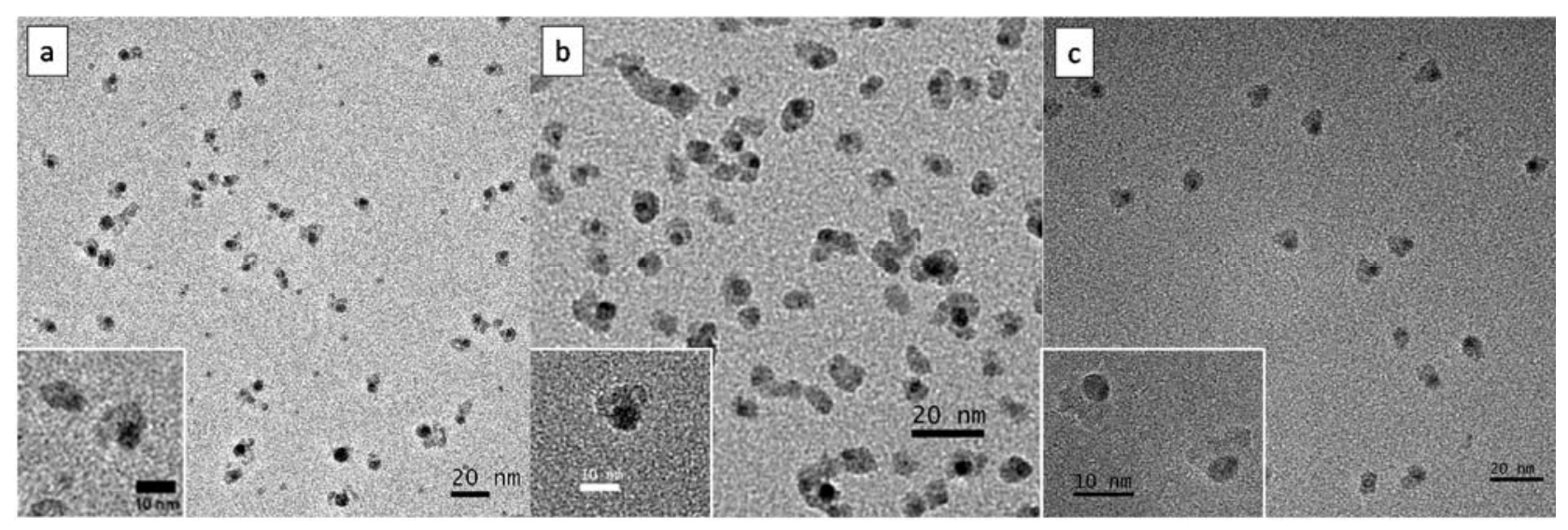

Figure 1 Low resolution TEM images of FeAg nanoparticles deposited onto a carbon membrane and left uncapped (a), embedded in silica (b) and in amorphous carbon (c). Higher magnification images of individual particles are shown in the inserts. The same "fried egg” structure is observed in all three cases. 
against oxidation and the samples are stable at ambient conditions. For iron-containing bi-metallic nanoparticles, the miscibility between iron and carbon has to be considered. In the size range discussed here (2-6 $\mathrm{nm}$ diameter) it has been shown for some elemental combinations (FeRh [39], FePt [40]) that even though carbon interstitials may be formed at the interface between metal particle and matrix, this carbide can be decomposed through heat treatment, leading to pure metallic, chemically ordered FePt and FeRh clusters. In other elemental combinations like FeCo a higher chemical affinity for carbide formation is observed and stable iron carbides are detected [41]. Whether such an intact nanoparticle structure with a well-defined interface can be achieved for the FeAg system and to which degree carbide formation will be an issue has so far not been addressed.

\subsection{Transmission Electron Microscopy}

Conventional Transmission Electron Microscopy (TEM) shows heterogeneous particles with a more or less spherical part surrounded by a more disordered structure, resembling a "nano-fried-egg". These heterostructures have been found for all three investigated systems: for uncapped nanoparticles deposited onto an ultrathin carbon membrane as well as for particles embedded in either silica or amorphous carbon matrices (cf. Fig. 1). The differences in contrast are due to differences in atomic number $Z$ and already hint towards a segregation of silver $(Z=47)$ in the spherical,

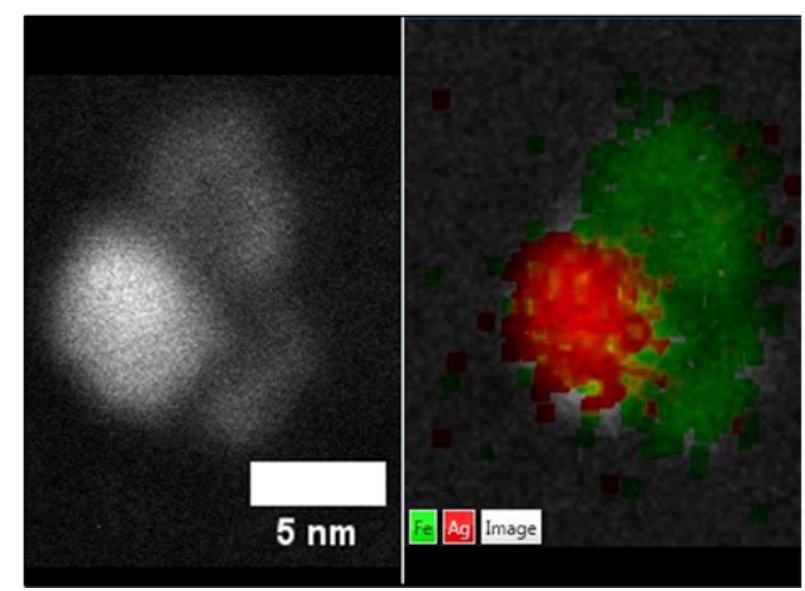

Figure 2 left: STEM-HAADF image of an FeAg nanoparticle in silica. Right: the EDX elemental mapping confirms the segregation into silver- and iron-containing parts. The used spectral lines are the $\mathrm{L} \alpha$ transition at $3 \mathrm{keV}$ for $\mathrm{Ag}$ and the $\mathrm{K} \alpha$ line at $6.4 \mathrm{keV}$ for Fe.

most contrasted part and iron $(Z=26)$ in the adjacent, less well defined one. This assumption is confirmed by Energy-dispersive $\mathrm{X}$-ray spectroscopy performed in Scanning Electron Microscopy (STEM-EDX) mode, as shown in figure 2 . Here the analysis of $x$-rays generated by the fast electrons allows for a spatially resolved elemental analysis in combination with an image collected in a High Angle Annular Dark Field (HAADF) detector.

In order to elucidate the crystalline structure and the chemical state of the FeAg nanoparticles we performed High Resolution TEM (HR-TEM). Depending

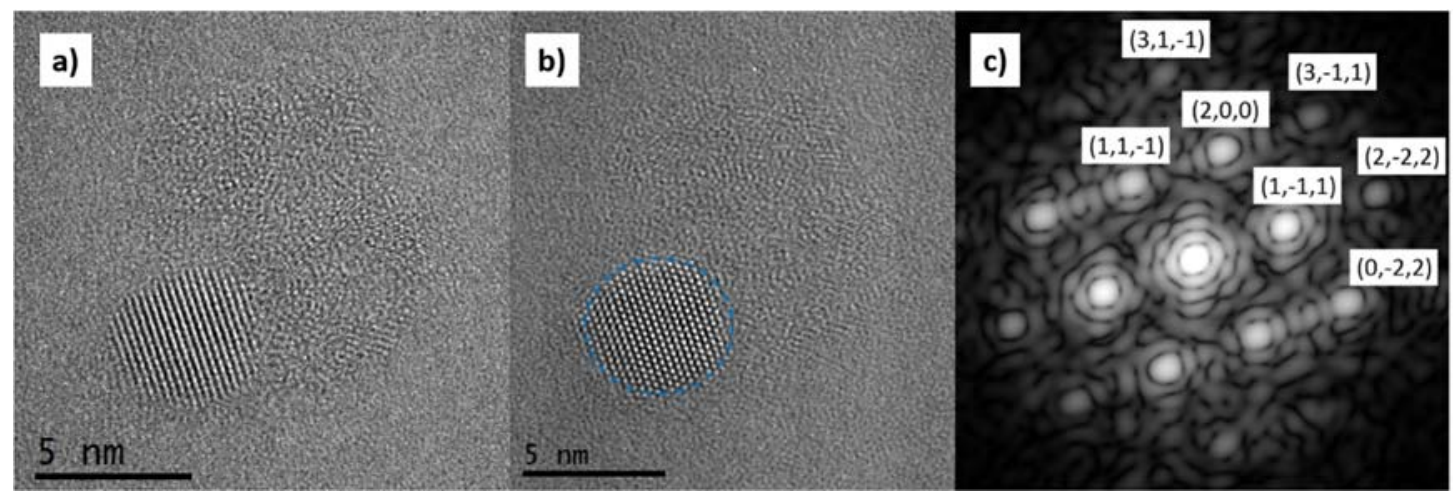

Figure 3 a) HR-TEM image of an FeAg nanoparticle in carbon. At the bottom left the spherical Ag part is visible, to the top and right of it the amorphous iron carbide can be discerned. b) Image of the same particle taken at different focalization conditions, here the crystalline structure of the silver sphere is more clearly visible but the top and right parts are difficult to distinguish from the carbon matrix. c) The FFT of the silver part (blue dashed line in b)) is in very good agreement with Ag fcc seen along the [110] direction. 


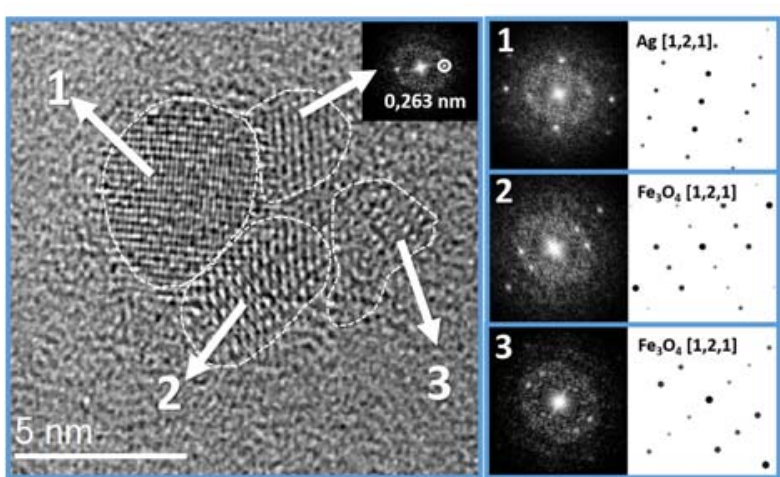

Figure 4 left: HR-TEM of an FeAg nanoparticle in silica; right: FFT patterns of the corresponding areas together with simulated patterns. Region 1 corresponds to Ag fcc seen along the $[1,2,1]$ axis, the FFT patterns of areas 2 and 3 agree with $\mathrm{Fe}_{3} \mathrm{O}_{4}$ along the $[1,2,1]$ direction. The only unambiguous distance in the top crystallite is also consistent with $\mathrm{Fe}_{3} \mathrm{O}_{4}$, as shown in the insert. In an image taken at different focal length a FFT pattern comparable to areas 2 and 3 was observed.

on the focusing conditions of the electron microscope it is possible to increase the contrast for either the silver- or the iron-containing part of the nanoparticles, as shown in figures $3 a$ and $b$.

For the spherical part of the particles crystalline atomic resolution is readily obtained for many particles (cf. figures 3 and 4) and the Fast Fourier Transform (FFT) patterns agree with metallic silver in the face-centred cubic (fcc) form, although sometimes twinned or distorted. The observed patterns are along the [110], [111], [112], [311] directions but also multiply twinned like decahedra or icosahedra are observed (cf. Electronic Supplementary Material). The fcc structure was evidenced down to $<2 \mathrm{~nm}$ diameter of the Ag part.

The iron-containing part, however, is more complicated to investigate. In no initially taken image a crystalline lattice was evidenced, these parts of the nanoparticles seem amorphous. Only after several minutes of electron irradiation one starts to see crystalline structures appear. The amorphous parts crystallize under the electron beam into several domains (cf. figure 4) and spots in the FFT patterns appear. In no case a clear evidence of metallic iron was found. For silica as matrix the observed structures agree with both magnetite $\left(\mathrm{Fe}_{3} \mathrm{O}_{4}\right)$ and hematite $\left(\alpha-\mathrm{Fe}_{2} \mathrm{O}_{3}\right)$ structures, the number of spots not being sufficient to discriminate between the two oxides. The comparison of the heats of formation of the different oxides, however, clearly favors the magnetite phase [42]. Diffraction patterns were simulated using the JEMS software [43]. For carbon as matrix only few spots were discernible after prolonged irradiation and no clear attribution to specific carbides or other non-metallic structures was possible. This means that the samples evolve under the influence of the electron beam. The initially amorphous low-contrast parts of the nanoparticles containing iron are meta-stable and altered whereas the silver nanospheres remain unchanged.

Note that in some cases the projected surface of the iron-containing part is large, as e.g. in figure 3. This suggests partial wetting of the surface by the iron fraction during chemical reaction and results in a "nanofried-egg" structure.

Selected Area Electron Diffraction (SAED) is a further tool for the averaged detection of interplanar distances. A large area (around 100-500 nm diameter) is irradiated by electrons and the diffracting distances result in spots and rings. This technique averages over hundreds of particles and is thus complementary to the single particle investigations discussed above. We have performed SAED measurements on $\mathrm{Fe}_{50} \mathrm{Ag}_{50}$ nanoparticles in both a-C and silica matrices, examples are shown in the Electronic Supplementary Material. All observed distances (five and four values, respectively) agree with those expected in fcc Ag or, for some distances, with bcc Fe, as shown in table S1. No distances of crystallized silver oxide or of iron oxides or carbides have been evidenced, corroborating the hypothesis of an amorphous iron-containing part of the nanoparticles.

\subsection{Optical Spectroscopy}

The optical properties of $\mathrm{Fe}_{50} \mathrm{Ag}_{50}$ nanoparticles were investigated through absorption spectroscopy after embedding in a transparent silica matrix. The measurements were performed using linearly polarized light (TM) and sample mounting at Brewster angle in order to prevent Fabry-Perot interferences within the sample film. As shown in fig. 5a, the localized surface plasmon resonance typical for silver nanoparticles [1] is broadened, damped and slightly red-shifted but still clearly visible. This observation is in qualitative 
agreement with the structural characterization of a spherical metallic silver particle in a heterogeneous environment of silicon- and iron-oxide. Note that the surface plasmon energy of silica-embedded nanoparticles is not size-dependent in this size range [44].

In order to verify the consistency of our assumed nanoparticle structure, we simulated the optical response of several test structures using a finite element method (commercial COMSOL software). All simulated particles have $5 \mathrm{~nm}$ diameter and are embedded in a medium with the experimentally determined refractive index of slightly porous silica $\left(\varepsilon_{m}=2.14\right)$. The spectra for the concentric geometries have also been calculated analytically using Mie theory [45]. The agreement between the obtained curves with the two simulation methods is excellent, thereby validating our approach.

Shown in fig. $5 \mathrm{~b}$ are simulated absorption spectra for different chemical compositions and geometries. The Fe@Ag core/shell structure, which we would expect from the thermodynamic considerations based on the surface tensions of iron and silver [24], has a predicted surface plasmon resonance at considerably higher energy than pure silver, we can rule out this geometry. Eccentric structures of a silver core in an iron oxide shell of varying stoichiometry, however, show the same broadened and red-shifted response as seen in the experiment. Even though we cannot infer the exact local composition of the iron oxide parts from our experiments, our simulations show that the presence of oxygen in the iron shell systematically red-shifts and broadens the silver plasmon resonance for any composition. Note that the same qualitative trend is observed for concentric or fully segregated Janus-type geometries and equally expected for structures deviating from a simple sphere. a: experiment

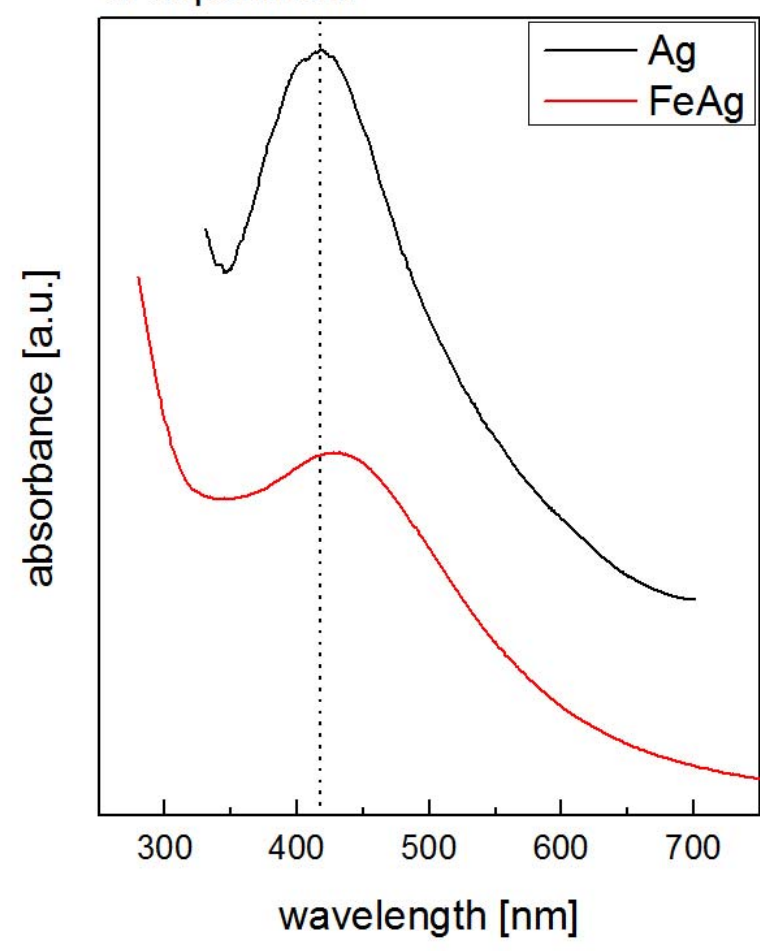

\section{b: simulation}

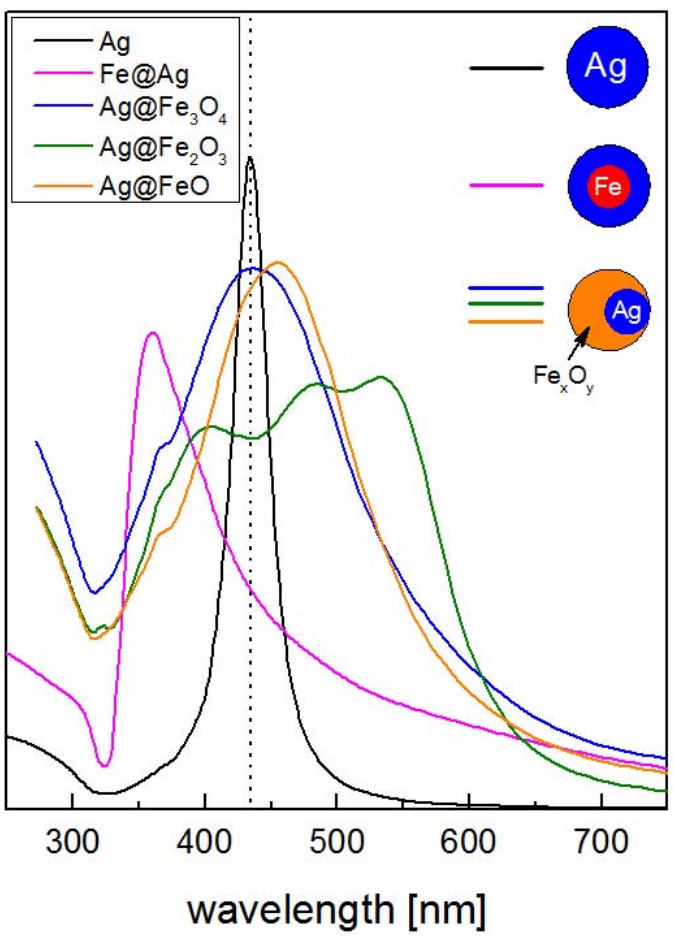

Figure 5: a) Optical absorption spectra of $\mathrm{Fe}_{50} \mathrm{Ag}_{50}$ nanoparticles (red, lower curve) with mean diameter of $4.5 \mathrm{~nm}$ and embedded in silica. The surface plasmon absorption peak for pure silver particles of $4.0 \mathrm{~nm}$ diameter in silica (black, upper curve) is shown for comparison. b) Simulated absorption spectra for five different compositions and geometries: pure silver, concentric Fe@Ag and eccentric Ag@Fe $\mathrm{O}_{\mathrm{y}}$ core shell structures. The vertical dotted line are guides to the eye and mark the position of the surface plasmon peak in pure silver. 
The optical measurements thus give evidence for i) the loss of the metallic character of iron, and ii) of chemical phase segregation between a pure silver and an iron oxide based dielectric domain, even if its exact morphology (centered or off-centered core-shell, Janus...) cannot be definitely inferred.

\subsection{Magnetic properties}

\section{X-ray Magnetic Circular Dichroism}

In order to learn more about the chemical composition of the iron-containing part of the FeAg nanoparticles and to see whether and to which degree the magnetic properties of iron are conserved we performed different magnetic experiments on carbon embedded FeAg nanoparticles. First of all we used X-ray Magnetic Circular Dichroism (XMCD) at the L2,3 edge of iron to derive the spin and angular momenta of the iron atoms. These experiments have been realized at the LNLS synchrotron in Campinas, Brazil.

Figure 6 displays X-ray absorption curves for the two photon helicities and the XMCD signal shows a clear magnetic contrast. The iron atoms retain a magnetic polarization even when combined to silver and embedded in an amorphous carbon matrix. Using the well-known sum rules [46] and a value $N_{h}=3.4$ for the number of holes in iron [47], we find a mean orbital momentum of $m_{L}=0.075 \mu_{B}$ and a mean spin moment of $m s=0.76 \mu_{B}$, resulting in a total moment of $0.83 \mu_{\text {B }}$. This value is significantly smaller than in bulk iron $\left(2.2 \mu_{B}\right)$ but clearly discernable.

A doublet is seen at the $L_{3}$ and, to a lesser degree, at the $L_{2}$ edge, the iron is not in its purely metallic form. The comparison with spectra in the literature allows the exclusion of predominant oxidation, all iron oxides, even in non-stoichiometric form and the nanoscale, display more complex X-ray Absorption Spectra and oscillating XMCD curves [48] due to a multiplet splitting. Amorphous iron carbides, on the other hand, show the same doublet as in our results [49] and by comparison we can estimate a composition around $\mathrm{Fe}_{30} \mathrm{C}_{70}$. We can thus identify the iron-containing parts of the nanoparticles, non-resolved in TEM, as an amorphous carbide, in agreement with the absence of stable crystalline structures in this carbon-rich region of the Fe-C phase diagram [50]. Note also that all stoichiometric iron carbides have reduced magnetic moments with respect to bulk iron of 1.7-1.8 $\mu_{\text {в }}$ per atom

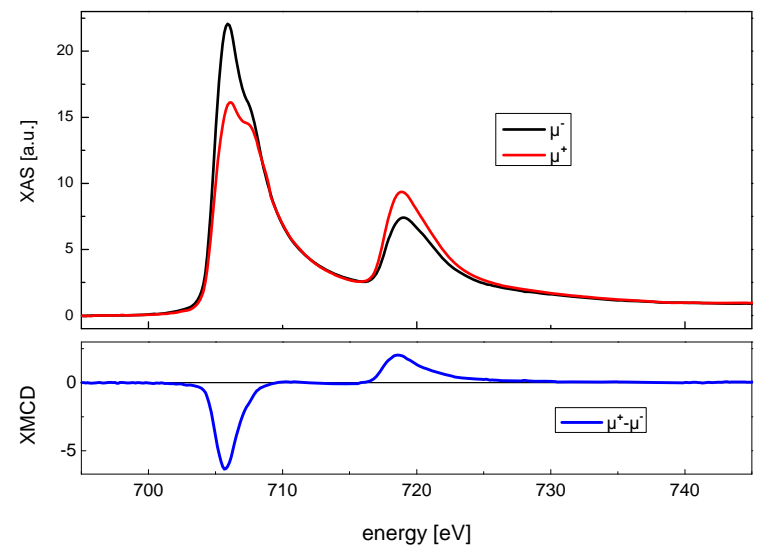

Figure 6 X-ray absorption (top) and XMCD (bottom) curves at the Fe $L_{2,3}$ edge acquired at $17 \mathrm{~K}$ and a magnetic field of $\mu_{0} H=4.5 \mathrm{~T}$.

[51] and we can expect even lower values for the amorphous state.

\section{SQUID magnetometry}

Further magnetic measurements were made with a SQUID magnetometer (MPMS XL5 from Quantum Design, USA). We performed a complete magnetic characterization combining namely zero-fieldcooled/field-cooled (ZFC/FC) susceptibility, magnetization $m(H, T)$ and low temperature Isothermal Remanent Magnetization (IRM) curves. We then fit the entire magnetic response curves simultaneously following the extended "triple fit" procedure, which has successfully been used in the past to treat and interpret the magnetic responses of both mono- and bi-metallic nanoparticles [52, 38, 53, 39, 54, 55].

This redundant multiple fitting uses only a very small number of fit parameters and thus allows obtaining reliable values for the magnetic diameter distribution and the magnetic anisotropy constant. The only adjustable parameters are the median particle size $D_{\text {mag }}$ with its dispersion in a log-normal distribution $w_{\text {mag, }}$ an effective anisotropy constant $K_{\text {eff }}$ with a dispersion in a Gaussian distribution $w_{K}$ and finally a possible biaxial component to the magnetic anisotropy described by the ratio of effective constants $K_{2} / K_{\text {eff. }}$. An amplitude parameter accounts for the number of particles in the sample but is not of interest in this study. Note that this procedure is highly sensitive to inter-particle interactions and thus very efficient for the exclusion of spurious deviations from the initial hypothesis of 

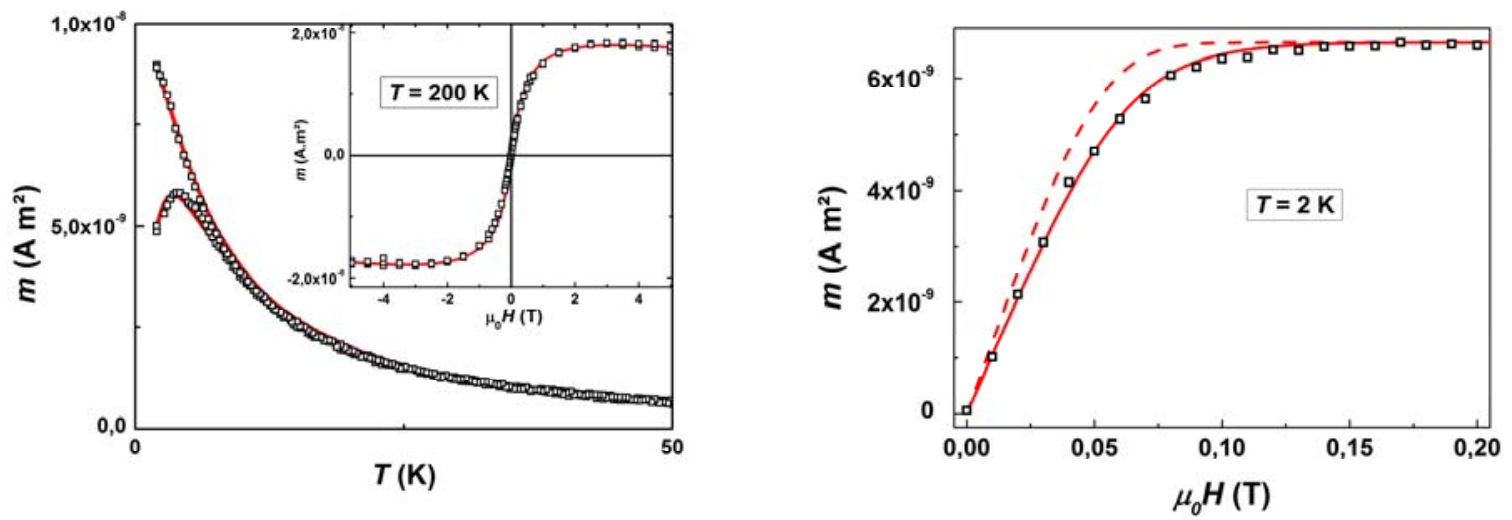

Figure 7: Magnetic characterization of $\mathrm{Fe}_{50} \mathrm{Ag}_{50}$ nanoparticles in amorphous carbon. Left: “triple fit”: experimental (points) ZFC/FC curves at $5 \mathrm{mT}$ and $m(H)$ at $\mathrm{T}=200 \mathrm{~K}$ (insert) together with fits (lines). Right: low temperature IRM data (points) and simulated response curves for uniaxial (dashed) and biaxial anisotropy with $K_{2} / K_{\text {eff }}=1$ (solid line).

non-interacting isolated macrospins.

Figure 7 shows the experimental magnetic data together with the obtained fits. In order to reduce the ambiguity for the obtained values from the fits even more, we use a median particle size derived from TEM. Since the iron-containing parts of the nanoparticles are rather ill defined, we determine the volume distribution for the well-contrasted spherical silver parts of the particles (cf. fig. 1 in the Electronic Supplementary Material) and, based on the 50/50 composition of the target rod, assume the same distribution for the number of iron atoms. With this value and using the magnetic moment per atom as obtained from $\mathrm{XMCD}$, we can nicely fit the experimental curves. The obtained values are $D_{\mathrm{mag}}=2.5 \mathrm{~nm}$ (from TEM), $w_{\mathrm{mag}}=$ $0.29 \pm 0.03, K_{\text {eff }}=38 \pm 5 \mathrm{~kJ} / \mathrm{m}^{3}, w_{K}=0.1 \pm 0.1$ and $K_{2} / K_{\text {eff }}$ $=1 \pm 0.5$. Figure $7 \mathrm{~b}$ shows clearly that a biaxial component to the magnetic anisotropy is necessary to reproduce the experimental data.

At this point we note that a different rough estimate of the size of the iron-containing parts is possible from the magnetic measurements. The IRM response, at least strictly at $0 \mathrm{~K}$, does not depend on the particle size but only on the saturation magnetization $M_{\text {sat }}$ [53]. The other magnetic curves shown above depend on the magnetic moment, i.e. $M_{\text {sat }}$ times volume. So it is possible to obtain identical curves in the "triple fit" with a doubled volume and half the saturation magnetization, describing a strongly dilute system as far as iron is concerned, such as in an alloy nanoparticle.
For this case the IRM curve is, however, impossible to reproduce and we can rule out this case.

\section{Discussion}

All experimental results for the physically prepared $\mathrm{Fe}_{50} \mathrm{Ag}_{50}$ nanoparticles are consistent and the overall image is as follows. The thermodynamic properties of the two metals are very different, the phase diagram shows no miscibility at any stoichiometry or temperature [20] and the surface tensions differ by a factor of two [24], suggesting a silver enrichment at the surface. Consequently the two metals segregate. Although we do not know the structure of the nanoparticles before deposition, we find the same Janus-type separation for all investigated environments. This observation hints towards an environment-independent Janustype segregation, in contrast to sequential fabrication by chemical methods $[13,27,28]$, where core-shell structures were observed. Possibly the chemical reaction of the iron with the environment changes the initial geometry and reverses the elemental order, as has been observed before [56]. So even though its high reactivity with the environment determines the chemistry of the iron moiety, the resulting structures are very comparable and we consequently discuss the results together.

The silver forms metallic, near-spherical nanocrystallites with fcc structure or sometimes multiply twinned decahedra or icosahedra, in agreement with 
previous observations [57, 58, 59].

The iron containing part of the particles readily reacts with the surrounding matrix forming metastable amorphous carbides or oxides. The latter can be transformed into multiply twinned crystallites under continuous irradiation in an electron microscope. The absence of crystallized Fe phases has already been observed in larger FeAg nanotruffles prepared by laser vaporization and functionalisation in a liquid [31]. The formation of disordered alloy domains as proposed in that article was, however, not observed in our case.

The silver nanospheres present a surface plasmon resonance in the blue part of the visible spectrum, in good agreement with the expected response for $\mathrm{Ag}$ nanoparticles in an oxide environment. It is broadened and shifted with respect to the reference peak of bare silver particles in silica, a trend that is well reproduced in numerical simulations of the optical response of eccentric $\mathrm{Ag} @ \mathrm{Fe}_{\mathrm{x}} \mathrm{O}_{\mathrm{y}}$ nanoparticles.

The iron fraction keeps a reduced, but significant magnetic moment despite the reaction with the carbon environment and the transformation into an amorphous carbide. All magnetic measurements can be reproduced by a small set of parameters and in agreement with the geometric characterization by electron microscopy. Both the magnetic moments per atom as well as the magnetic anisotropy are strongly reduced with respect to the iron bulk and a strong biaxial component to the magnetic anisotropy is observed. All this is in accordance with the strongly heterogeneous shapes and the amorphous character of the iron carbide.

Despite this combination of both properties in the same particle, the iron-silver system in particular does not seem the most adequate candidate system for magneto-plasmonic studies due to the high reactivity of the exposed iron fraction and the resulting reduction of magnetism and the strong phase separation. For other iron-containing nanoparticles, fabricated by the same preparation method (FeRh [39] and FePt [40]), chemically and thermally stable alloy particles have been obtained but here the two metals are miscible in the bulk phase. For iron and gold, with their very limited macroscopic miscibility, a size-induced miscibility was reported in nanoparticles smaller than $10 \mathrm{~nm}$ [60] and for different stoichiometries [61]. The miscibility, macroscopic or nanoscale-induced, of iron in the other metal is the key for avoiding chemical reactions and thus the degradation of the particles. If iron and silver are to be used in magneto-plasmonic nanoparticles, then either the particles have to be protected by an adequate ligand shell or the miscibility would have to be induced by e.g. a specific environment or temperature/pressure treatments. The protection of a pure iron particle by sequential deposition of a protective silver shell as demonstrated in [62] could be another approach.

Finally we would like to comment on the reliability of the interpretation of the magnetic data. Due to the comparably large diameter dispersion and the ill definition of the amorphous iron containing structures the magnetic response curves are quite ambiguous. It is only after separate determination of some of the input parameters (magnetic moment per atom from XMCD and median particle size from TEM) that an unambiguous set of fit parameters was obtained. A conservative estimate of the relative errors of the values stated above of the order of $10 \%$ quickly rises to well above $100 \%$ if the median size or the saturation magnetization are left as free fit parameters. This underlines the importance of the redundant fitting of several entire curves as done in the "triple fit" procedure, together with complementary experiments.

\section{Conclusion}

We have successfully fabricated surfactant-free small $\mathrm{Fe}_{50} \mathrm{Ag}_{50}$ nanoparticles by a physical route. Multiply twinned particles with heterogeneous phases are evidenced. We demonstrate the coexistence of both metals in the same nanoparticles which consequently display both plasmonic and magnetic responses. The metals segregate completely in all investigated systems. The silver fraction is always found in metallic, fcc crystallized or sometimes multiply twinned nanospheres, while the iron forms amorphous structures that react with the surrounding matrix to form carbides or oxides. These iron compounds are meta-stable and crystallize under the electron beam of a TEM. The $\mathrm{Fe}_{50} \mathrm{Ag}_{50}$ nanoparticles have a broadened but clearly discernable plasmon resonance with respect to bare silver particles. The magnetic moment of the iron part is quantified as reduced with respect to the bulk values of pure $\mathrm{Fe}$ and its oxides, a reduction we attribute to the presence of carbides and the amorphous 
structure. The derived biaxial magnetic anisotropy is low, in accordance with the highly heterogeneous structures observed in electron microscopy, but possible to quantify.

In conclusion we successfully demonstrate the advanced experimental techniques necessary for the unambiguous characterization of complex bi-metallic nanoparticles comprising both plasmonic and magnetic responses.

\section{Acknowledgements}

Financial support through a "Chaire Française dans l'État de São Paulo" and from the São Paulo Research Foundation (FAPESP, 2013/14262-7 and 16/12807-4) for M.H., from the Science Without Borders "Special Visiting Scientist" program, contract number 88881.030488/2013-01, and from the Region RhôneAlpes in the frame of an ARC (Academic Research Community) doctoral grant for J.R. is gratefully acknowledged. This work was performed using the Lyon Cluster Research Platform PLYRA, the Lyon Center for Microscopy CLYM, the Lyon Center for Magnetometry CML and at the Brazilian Nanotechnology National Laboratory (LNNano). The Laboratório Nacional de Luz Síncrotron (LNLS, Campinas, Brazil) is thanked for the use of the PGM beamline. We gratefully acknowledge technical support from C. Albin, C. Clavier and N. Blanchard in Lyon and from A. de Siervo, J. Bettini and J. Cezar in Campinas.

Electronic Supplementary Material: Supplementary material with more information on the determined size distribution and example TEM images is available in the online version of this article at http://dx.doi.org/10.1007/s12274-*******_* (automatically inserted by the publisher).

\section{References}

[1] Kreibig, U. and Vollmer, M. Optical properties of metal clusters. Springer series in materials science. Springer Berlin, (1995).

[2] Coey, J. M. D. Magnetism and Magnetic Materials. Cambridge University Press, (2010).
[3] Alloyeau, D., Mottet, C., and Ricolleau, C., editors. Nanoalloys: Synthesis, Structure and Properties. Springer-Verlag London, (2012).

[4] Calvo, F., editor. Nanoalloys: From Fundamentals to Emergent Applications. Elsevier, (2013).

[5] Ferrando, R. Structure and Properties of Nanoalloys, volume 10 of Frontiers of Nanoscience. Elsevier, (2017).

[6] García, S., Zhang, L., Piburn, G. W., Henkelman, G., and Humphrey, S. M. ACS Nano 8, 11512 (2014).

[7] Sotiriou, G. A., Visbal-Onufrak, M. A., Teleki, A., Juan, E. J., Hirt, A. M., Pratsinis, S. E., and Rinaldi, C. Chem. Mater. 25, 4603 (2013).

[8] Armelles, G., Cebollada, A., García-Martín, A., and González, M. U. Adv. Opt. Mater. 1, 10 (2013).

[9] Peng, S., Lei, C., Ren, Y., Cook, R. E., and Sun, Y. Angew. Chem. Int. Ed. 50, 3158 (2011).

[10] Hao, R., Xing, R., Xu, Z., Hou, Y., Gao, S., and Sun, S. Adv. Mater. 22, 2729 (2010).

[11] Wang, J., Wu, X., Wang, C., Shao, N., Dong, P., Xiao, R., and Wang, S. ACS Appl. Mater. Interfaces 7(37), 20919-20929 (2015).

[12] Bogani, L., Cavigli, L., de Julián Fernández, C., Mazzoldi, P., Mattei, G., Gurioli, M., Dressel, M., and Gatteschi, D. Adv. Mater. 22, 4054 (2010).

[13] Lu, L., Zhang, W., Wang, D., Xu, X., Miao, J., and Jiang, Y. Mater. Lett. 64, 1732 (2010).

[14] Moscoso-Londoño, O., Muraca, D., Tancredi, P., CosioCastañeda, C., Pirota, K. R., and Socolovsky, L. M. J. Phys. Chem. C 118, 13168 (2014).

[15] Mahmoudi, M. and Serpooshan, V. ACS Nano 6, 2656 (2012).

[16] Lin, A. Y., Young, J. K., Nixon, A. V., and Drezek, R. A. Small 10, 3246 (2014).

[17] Han, X. X., Schmidt, A. M., Marten, G., Fischer, A., Weidinger, I. M., and Hildebrandt, P. ACS Nano 7, 3212 (2013).

[18] Wang, H., Shen, J., Li, Y., Wei, Z., Cao, G., Gai, Z., Hong, K., Banerjee, P., and Zhou, S. ACS Appl. Mat. Interfaces 5, 9446 (2013).

[19] Andreazza, P., Pierron-Bohnes, V., Tournus, F., AndreazzaVignolle, C., and Dupuis, V. Surf. Sci. Rep. 70, 188 (2015).

[20] Swartzendruber, L. J. Bull. Alloy Phase Diag. 5, 560 (1984).

[21] Kataoka, N., Sumiyama, K., and Nakamura, Y. J. Phys F: Met. Phys. 18, 1049 (1988).

[22] Wan, H., Tsoukatos, A., Hadjipanayis, G. C., Li, Z. G., and Liu, J. Phys. Rev. B 49, 1524 (1994).

[23] Sakurai, M., Makhlouf, S., Sumiyama, K., Wakoh, K., and Suzuki, K. Jap. J. Appl. Phys. 33, 4090 (1994).

[24] Yaws, C. L. Chemical properties handbook: physical, thermodynamic, environmental, transport, safety, and health related properties for organic and inorganic chemicals. McGraw-Hill, New York, (1999).

[25] Ferrando, R., Jellinek, J., and Johnston, R. L. Chem. Rev. 108, 845 (2008).

[26] Pellarin, M., Issa, I., Langlois, C., Lebeault, M.-A., Ramade, 
J., Lermé, J., Broyer, M., and Cottancin, E. J. Phys. Chem. C 119, 5002 (2015).

[27] Carroll, K. J., Hudgins, D. M., Spurgeon, S., Kemner, K. M., Mishra, B., Boyanov, M. I., Lester W. Brown, I., Taheri, M. L., and Carpenter, E. E. Chem. Mater. 22, 6291 (2010).

[28] Wang, L., Yang, K., Clavero, C., Nelson, A. J., Carroll, K. J., Carpenter, E. E., and Lukaszew, R. A. J. Appl. Phys. 107, 09B303 (2010).

[29] Luo, S., Yang, S., Wang, X., and Sun, C. Chemosphere 79, 672 (2010).

[30] Markova, Z., Siskova, K. M., Filip, J., Cuda, J., Kolar, M., Safarova, K., Medrik, I., and Zboril, R. Environ. Sci. Technol. 47, 5285 (2013).

[31] Amendola, V., Scaramuzza, S., Agnoli, S., Granozzi, G., Meneghetti, M., Campo, G., Bonanni, V., Pineider, F., Sangregorio, C., Ghigna, P., Polizzi, S., Riello, P., Fiameni, S., and Nodari, L. Nano Res. 8, 4007 (2015).

[32] Scaramuzza, S., Badocco, D., Pastore, P., Coral, D. F., Fernández van Raap, M. B., and Amendola, V. ChemPhysChem 18(9), 1026-1034 (2017).

[33] Andrews, M. P. and O’Brien, S. C. J. Phys. Chem. 96, 8233 (1992).

[34] Perez, A., Dupuis, V., Tuaillon-Combes, J., Bardotti, L., Prevel, B., Bernstein, E., Mélinon, P., Favre, L., Hannour, A., and Jamet, M. Adv. Eng. Mater. 7, 475-485 (2005).

[35] Rousset, J. L., Cadrot, A. M., Cadete Santos Aires, F. J., Renouprez, A., Mélinon, P., Perez, A., Pellarin, M., Vialle, J. L., and Broyer, M. J. Chem. Phys. 102, 8574 (1995).

[36] Tournus, F., Blanc, N., Tamion, A., Hillenkamp, M., and Dupuis, V. Phys. Rev. B 81, 220405 (2010).

[37] Tournus, F., Blanc, N., Tamion, A., Dupuis, V., and Epicier, T. J. Appl. Phys. 109, 07B722 (2011).

[38] Tamion, A., Hillenkamp, M., Hillion, A., Tournus, F., Tuaillon-Combes, J., Boisron, O., Zafeiratos, S., and Dupuis, V. J. Appl. Phys. 110, 063904 (2011).

[39] Hillion, A., Cavallin, A., Vlaic, S., Tamion, A., Tournus, F., Khadra, G., Dreiser, J., Piamonteze, C., Nolting, F., Rusponi, S., Sato, K., Konno, T. J., Proux, O., Dupuis, V., and Brune, H. Phys. Rev. Lett. 110, 087207 (2013).

[40] Dupuis, V., Khadra, G., Linas, S., Hillion, A., Gragnaniello, L., Tamion, A., Tuaillon-Combes, J., Bardotti, L., Tournus, F., Otero, E., Ohresser, P., Rogalev, A., and Wilhelm, F. J. Magn. Magn. Mater. 383, 73 (2015).

[41] Dupuis, V., Robert, A., Hillion, A., Khadra, G., Blanc, N., Roy, D. L., Tournus, F., Albin, C., Boisron, O., and Tamion, A. Beilstein J. Nanotechnol. 7, 1850 (2016).

[42] Navrotsky, A., Ma, C., Lilova, K., and Birkner, N. Science 330, 199-201 (2010).

[43] http://www.jems saas.ch/.
[44] Hillenkamp, M., Di Domenicantonio, G., Eugster, O., and Félix, C. Nanotechnology 18, 015702 (2007).

[45] Cottancin, E., Broyer, M., Lermé, J., and Pellarin, M. Handbook of Nanophysics: Nanoelectronics and Nanophotonics, chapter Optical Properties of Metal Clusters and Nanoparticles. CRC Press, Boca Raton, FL, USA (2011).

[46] Chen, C. T., Idzerda, Y. U., Lin, H.-J., Smith, N. V., Meigs, G., Chaban, E., Ho, G. H., Pellegrin, E., and Sette, F. Phys. Rev. Lett. 75, 152-155 (1995).

[47] Sipr, O. and Ebert, H. Phys. Rev. B 72, 134406 Oct (2005).

[48] Pellegrin, E., Hagelstein, M., Doyle, S., Moser, H. O., Fuchs, J., Vollath, D., Schuppler, S., James, M. A., Saxena, S. S., Niesen, L., Rogojanu, O., Sawatzky, G. A., Ferrero, C., Borowski, M., Tjernberg, O., and Brookes, N. B. phys. status solidi (b) 215, 797 (1999).

[49] Furlan, A., Jansson, U., Lu, J., Hultman, L., and Magnuson, M. J. Phys.: Condens. Matter 27, 045002 (2015).

[50] Okamoto, H. J. Phase Equil. 13(5), 543-565 (1992).

[51] Hofer, L. J. E. and Cohn, E. M. J. Am. Chem. Soc. 81, 1576 (1959).

[52] Tamion, A., Hillenkamp, M., Tournus, F., Bonet, E., and Dupuis, V. Appl. Phys. Lett. 95, 062503 (2009).

[53] Hillion, A., Tamion, A., Tournus, F., Gaier, O., Bonet, E., Albin, C., and Dupuis, V. Phys. Rev. B 88, 094419 (2013).

[54] Oyarzún, S., Tamion, A., Tournus, F., Dupuis, V., and Hillenkamp, M. Sci. Rep. 5, 14749 (2015).

[55] Dupuis, V., Khadra, G., Hillion, A., Tamion, A., TuaillonCombes, J., Bardotti, L., and Tournus, F. Phys. Chem. Chem. Phys. 17, 27996 (2015).

[56] Tao, F., Grass, M. E., Zhang, Y., Butcher, D. R., Renzas, J. R., Liu, Z., Chung, J. Y., Mun, B. S., Salmeron, M., and Somorjai, G. A. Science 322(5903), 932-934 (2008).

[57] Li, C., Lu, N., Xu, Q., Mei, J., Dong, W., Fu, J., and Cao, Z. J. Cryst. Growth 319(1), 88 - 95 (2011).

[58] Volk, A., Thaler, P., Koch, M., Fisslthaler, E., Grogger, W., and Ernst, W. E. J. Chem. Phys. 138(21), 214312 (2013).

[59] Barke, I., Hartmann, H., Rupp, D., Fluckiger, L., Sauppe, M., Adolph, M., Schorb, S., Bostedt, C., Treusch, R., Peltz, C., Bartling, S., Fennel, T., Meiwes-Broer, K.-H., and Möller, T. Nat. Comm. 6, 6187 (2015).

[60] Mukherjee, P., Zhang, Y., Kramer, M. J., Lewis, L. H., and Shield, J. E. Appl. Phys. Lett. 100, 211911 (2012).

[61] Mukherjee, P., Manchanda, P., Kumar, P., Zhou, L., Kramer, M. J., Kashyap, A., Skomski, R., Sellmyer, D., and Shield, J. E. ACS Nano 8(8), 8113-8120 (2014).

[62] Binns, C., Qureshi, M. T., Peddis, D., Baker, S. H., Howes, P. B., Boatwright, A., Cavill, S. A., Dhesi, S. S., Lari, L., Kröger, R., and Langridge, S. Nano Lett. 13(7), 3334-3339 (2013). 